\title{
ANÁLISE DOS CONCEITOS DE NEGOCIAÇÃO/ACOMODAÇÃO DA TEORIA DE M. LEININGER ${ }^{1}$
}

\author{
Astrid Eggert Boehs ${ }^{2}$
}

Boehs AE. Análise dos conceitos de negociação/acomodação da teoria de M. Leininger. Rev Latino-am Enfermagem 2002 janeiro-fevereiro; 10(1):90-6.

Este trabalho tem por objetivo clarificar o modo de implementação do cuidado negociação/acomodação, preconizado pela teoria da Universalidade e Diversidade do Cuidado de Madeleine Leininger. Tem como recurso a técnica de análise de conceito para fazer análise na literatura. Verificou-se que o conceito de acomodação define a idéia de enquadramento, de algo passivo, enquanto na negociação cada parte leva recursos e necessidades a serem satisfeitas. Nos trabalhos analisados que utilizaram a teoria de Leininger, apenas um demonstra aproximar-se da negociação. Como conclusão, considera-se que acomodação é diferente de negociação, sendo que na prática ocorre com mais ênfase a acomodação do que a negociação.

DESCRITORES: negociação, enfermagem transcultural, teoria de enfermagem

\section{AN ANALYSIS OF THE NEGOTIATION/ACCOMODATION CONCEPTS IN M. LEININGER'S THEORY}

This work aims at clarifying the method of implementation of the negotiation/accommodation care preconized by Madeleine Leininger's theory concerning the Universality and Diversity of Care. The conceptual analysis technique is used as a tool to analyze the literature. It was verified that the concept of accommodation defines the idea of adaptation, of something passive, whilst in negotiation, each party commands resources and needs to be fulfilled. Among the different works which were analyzed and used Leininger's theory, only one showed to approach negotiation. As a conclusion, accommodation is considered to be different from negotiation, the former being more commonly seen in practice than the latter.

KEY WORDS: negotiation, transcultural nursing, nursing theory

\section{ANÁLISIS DE LOS CONCEPTOS NEGOCIACIÓN/ACOMODACIÓN DE LA TEORÍA DE M. LEININGER}

Este trabajo tiene por objetivo clarificar el modo de implementación del cuidado negociación/acomodación preconizado por la teoría de la universalidad y diversidad del cuidado de Madeleine Leininger. Como recurso se utilizó la técnica de análisis de concepto para hacer análisis en la literatura. Se verificó que el concepto de acomodación define la idea de encuadramiento de algo pasivo, mientras que en la negociación, cada parte posee elementos y necesidades a ser satisfechas. En los estudios analizados que utilizaron la teoría de Leininger, solo uno demuestra aproximarse a la negociación. Como conclusión se considera que la acomodación es diferente de la negociación, siendo que en la practica clínica ocurre con mayor énfasis la acomodación más que la negociación.

DESCRIPTORES: negociación, enfermería transcultural, teoría, enfermería

\footnotetext{
${ }^{1}$ Trabalho final da disciplina Concepções Teóricas da Enfermagem do Curso de Doutorado em Filosofia da Enfermagem, Pós-Graduação de Enfermagem da Universidade Federal de Santa Catarina; ${ }^{2}$ Docente do Departamento de Enfermagem e Doutoranda do Curso de Doutorado em Filosofia da Enfermagem da Universidade Federal de Santa Catarina, e-mail: astridp@nfr.ufsc.br
} 
INTRODUÇÃO E OBJETIVO

$\boldsymbol{O}_{\text {fator cultural na saúde/doença, antes considerado fator }}$ secundário, recentemente tem começado a receber mais atenção dos profissionais da área de saúde e dos cientistas sociais. A maioria dos mentores de programas de saúde parte do pressuposto de que a informação gera transformação automática ${ }^{(1)}$. Por outro lado, os profissionais, principalmente aqueles que se preocupam com a prevenção, foram aos poucos constatando que não basta somente colocar serviços à disposição, e sim fazer a clientela utiliza-los, revelando dessa forma que o cliente faz sua própria valoração e decide sobre a conveniência da utilização dos serviços. 0 estudo dos fatores culturais na saúde e doença têm, assim, uma importante contribuição a oferecer.

Nas últimas décadas, autores da enfermagem, em diferentes países, também têm se preocupado em relação ao cuidado e aos fatores culturais. Madeleine Leininger, na década de 50, ao trabalhar num lar de crianças provenientes de diversas nacionalidades, verificou que as mesmas queriam ser cuidadas de maneiras diferentes. Desenvolveu, então, a teoria transcultural do cuidado, na qual considera que o cuidado ao ser humano é universal, isto é, o ser humano, para nascer, crescer, manter sua vida e morrer, precisa ser cuidado, porém cada cultura, de acordo com seu ambiente e estrutura social, terá sua própria visão de saúde, doença e cuidado. Para a autora, existe um sistema profissional de cuidado e cura que é o sistema organizado, formalmente reconhecido, e oferecido pelos profissionais de saúde. Neste sistema profissional, considera-se que 0 cuidado é a essência da enfermagem. Além disso, ainda há 0 sistema popular que é o sistema local, onde se inclui a família. A saúde para essa autora, é o estado percebido ou cognitivo de bemestar, que capacita o indivíduo ou grupo a efetuar as atividades segundo os padrões desejados em determinada cultura. Cultura são os valores, crenças e práticas compartilhadas, apreendidas ao longo das gerações.

Foram propostas três formas para realizar o cuidado, levando em conta 0 modo de vida e crenças compartilhadas: preservação do cuidado, acomodação e repadronização(2). Preservação se constitui naqueles cuidados já praticados por um indivíduo, família ou grupo, benéficos ou mesmo inócuos para a saúde. Acomodação são ações e decisões para assistir, dar suporte, facilitar as pessoas de uma determinada cultura a adaptar-se ou negociar com provedores de saúde profissionais. Repadronização são ações e decisões para facilitar, dar suporte, que ajudam indivíduos, grupos a reordenar, trocar ou em grande parte modificar seus modos de vida para o novo, o diferente, beneficiando os padrões de cuidado à saúde. Posteriormente acrescenta à palavra "acomodação" a palavra "negociação" mantendo, no entanto a explicação das publicações anteriores. Da mesma forma, à "repadronização" ela acrescenta a palavra "reestruturação"(3).

Minha experiência com a teoria de Leininger ocorreu primeiramente no trabalho de dissertação de mestrado ${ }^{(4)}$ com famílias de recém-nascidos e depois no cotidiano de um Centro de Saúde de periferia urbana, bem como na orientação ou acompanhamento de trabalhos de conclusão de curso de graduação e especialização. Estas experiências possibilitaram visualizar que, sendo uma grande teoria, há questões que precisam ser aproximadas da prática. Há dúvidas, como levar em conta os fatores culturais utilizando princípios da antropologia de não intervenção, mas ao mesmo tempo, ser profissional de enfermagem que se constitui numa profissão que tem como princípio intervir. Especialmente o que tem chamado atenção é o entendimento da acomodação/negociação, uma vez que Leininger, ao colocar as duas palavras de forma conjunta, mantém a mesma explicação para ambas. No entanto, em várias discussões com alunos e profissionais, levantou-se o questionamento: acomodar cuidado é realmente o mesmo que negociar cuidado?

Considerando esta questão, pretendo fazer a análise dos termos acomodação e negociação do cuidado e da inserção destes termos em trabalhos de pesquisa e trabalhos de implementação da teoria de Leininger na assistência.

\section{ANÁLISE DE CONCEITOS}

O desenvolvimento de conceitos é um estágio importante no progresso da disciplina de enfermagem. Processos usados no desenvolvimento de conceitos receberam considerável atenção das estudiosas da enfermagem durante as últimas décadas ${ }^{(5-7)}$.

Neste trabalho, procuro situar a análise tentando clarificar a negociação e a acomodação, utilizando algumas fases do método de análise de conceito ${ }^{(6)}$. Procurei adaptar a forma de análise à minha própria forma e entendimento e às características dos dados disponíveis. Além disso, a leitura de outros autores ${ }^{(5,7)}$ de alguma forma circundaram as idéias desta análise. Dessa maneira, segui o seguinte itinerário:

- seleção das fontes de consulta;

- identificação do conceito de negociação e acomodação em dicionários e literatura; da área de administração;

- identificação da negociação/acomodação de acordo com diversos autores; da enfermagem transcultural;

- identificação dos atributos dos conceitos com base na literatura acima mencionada;

- apresentação dos antecedentes e conseqüências do conceito; - oposição aos conceitos de negociação e de acomodação. 


\section{TENTANDO CLARIFICAR A NEGOCIAÇÃOI ACOMODAÇÃO}

Fontes de consulta

A literatura selecionada refere-se aos principais dicionários da língua portuguesa, bem como obras na área de administração $e$ trabalhista onde este fenômeno é analisado com mais frequêencia. Esta literatura foi buscada via computador em bibliotecas especializadas na área de administração em Florianópolis. Segundo a literatura sobre enfermagem transcultural ${ }^{(3)}$ foram apresentadas as diferentes pesquisas realizadas. Além disso, foram consultadas publicações periódicas brasileiras referentes a 1997 e 1998 (Revista Latino-Americana de Enfermagem; Revista da Escola de Enfermagem da USP e Revista Brasileira de Enfermagem), bem como revistas de língua inglesa (Research in Nursing \& Health, ano 1997; Sholarly Inquiry for Nursing Practice, ano de 1997; e Journal Advanced Nursing, 1997 e 1998). Foram resgatadas na Pós-Graduação de Enfermagem da UFSC as dissertações de mestrado e monografias de especialização que tiveram como tema a aplicação de referencial teórico baseado na teoria de Leininger.

Identificação de negociação e acomodação sob o ponto de vista geral

A negociação é o ato ou efeito de negociar, contrato, ajuste, discussão de um assunto de interesse comum entre agentes autorizados $^{(8)}$. Com base nos conhecimentos de administração, economia e filosofia ${ }^{\left({ }^{9}\right)}$, negociação apresenta-se como uma interação verbal em que as partes propõem, contrapõem e argumentam. Visa um resultado que é um acordo, uma determinada proposição prática que recebe 0 assentimento das partes envolvidas, enfim visa solucionar divergências. E esse autor entende divergência entendido como possuir objetivos diferentes ou concepções diversas, ou mesmo quando duas pessoas almejam algo impossível de ser partilhada. Há diversos tipos de negociação: trabalhista, diplomática, comercial, administrativa, política. Há também, uma realidade multifacética das negociações:

Face lógico-lingüística: as partes argumentam, apresentam provas e cada parte formula para si hipóteses de resultado;

Face psicológica: cada parte tem percepções e expectativas de si e do outro, que vão mudando à medida que a negociação vai se desenrolando.

Face política - não está relacionada à política propriamente dita, e sim refere-se ao poder, isto é, ao poder de barganha das partes. A negociação é um jogo de forças em que o poder está presente, como uma realidade circundante que pode auxiliar ou destruir o processo de negociação;
Face social - a negociação sempre envolve grupos e papéis de uma determinada sociedade nos quais entram em jogo o poder dos envolvidos;

Face econômica - mesmo que indiretamente, esta questão está relacionada;

Face antropológica: envolve rituais com regras e cerimoniais. Acham-se integrados nos usos e costumes de sociedade, constituindo em determinadas culturas o que é e o que não é negociável;

Face administrativa: é uma face ritual onde ocorre planejamento, organização, coordenação e decisão;

Face tácito-estratégica: durante a negociação há manobras, artimanhas para conduzir a outra parte a uma decisão.

Ainda segundo este autor, há a estrutura e o processo de negociação. A estrutura é o conjunto de relações que é o resultado da interação das características das partes. Cada parte entra na negociação com necessidades insatisfeitas que o outro possa atender, bem como com recursos para oferecer. Se apenas uma das partes possuir as alternativas, não há negociação e, sim, imposição. 0 processo, por sua vez, é um tipo de interação em que as partes em condições semelhantes buscam resolver diferenças na obtenção de um acordo. É essencialmente verbal, e o negociador precisa encontrar meios para alterar a expectativa do outro.

Quanto ao termo acomodação, é: 1) ato de acomodar; 2) alojamento, aposentos; 3) adaptação; 4) conforto, comodidade; 5) todo processo que visa a solução de conflito ${ }^{(8)}$.

Identificação da negociação/acomodação de acordo com a literatura da enfermagem transcultural

Referenciais teóricos baseados na teoria de Leininger e aplicados na prática

A autora da dissertação de mestrado ${ }^{(10)}$ que utilizou 0 referencial teórico ${ }^{(2)}$ para famílias com adolescentes grávidas oriundas de áreas da periferia urbana, bem como de estrato médio, afirma que, nas situações em que não é possível preservar o cuidado, será feita a acomodação, isto é, a união dos modos de cuidar do enfermeiro com aqueles do cliente. Esta união se faz através da negociação interacional. Esta negociação seria uma forma de relação para não gerar a imposição. A autora apresenta um exemplo: à mãe que colocava a faixa abdominal no recém-nascido, foi recomendado que ela poderia continuar utilizando-a, mas de uma forma que ficasse mais frouxa.

Em estudo ${ }^{(4)}$ com famílias de recém-nascidos de origem açoriana, residentes na ilha de Santa Catarina, a autora não se refere à negociação e sim à acomodação, que entendeu ser a conjugação 
de dois tipos de cuidado quando a percepção da família é diferente do profissional. Para fazer esta conjugação, utilizou os componentes do conceito de cuidar que são: - auxiliar, trocar idéias, compreender, sugerir ações, fazer técnicas, fazer junto, reforçar, facilitar e outros que também foram utilizados para realizar a preservação e repadronização do cuidado.

Em outra dissertação de mestrado ${ }^{(11)}$ foi utilizado o referencial teórico ${ }^{(2)}$ na assistência tanto no ambulatório como no domicilio de famílias nas quais havia um ou mais membros com Aids (Síndrome da Imunodeficiência Adquirida). Utilizou a acomodação do cuidado que é realizada quando há divergência entre o cuidado profissional e o popular. A autora não explicitou no seu trabalho, como realizou a acomodação do cuidado.

No relato de experiência ${ }^{(12)}$ com base na aplicação do referencial teórico ${ }^{(2)}$ na assistência a uma família migrante, onde seus componentes eram todos desempregados, vivendo de esmolas, residentes em área de invasão na periferia urbana, a assistência foi realizada exclusivamente no domicílio. Como resultado, as autoras apontam a grande dificuldade e as raras oportunidades de realizar a acomodação.

Outro estudo ${ }^{(13)}$ fundamentado no referencial teórico ${ }^{(3)}$, está relacionado a assistência a mulheres quando do nascimento de seus primeiros filhos na maternidade e no domicílio. Seu foco foram os rituais de cuidado, que podem ser acomodados ou negociados pela enfermeira. Esta acomodação/negociação refere-se àquelas ações explícitas que ajudam as pessoas de uma determinada cultura a adaptarem ou negociarem os ritos no sentido de obter resultados mais benéficos. A autora mostrou que, ao atuar acomodando, negociou, isto é, buscou entendimento com as mulheres sobre a melhor maneira de proceder as alterações dos ritos de cuidado. Procurava nesta negociação garantir que houvesse uma forma participativa através do diálogo numa ação-reflexão-ação, partindo das experiências que as mulheres possuíam, valorizando seus conhecimentos e práticas. Fez uma síntese, dizendo que, para acomodar, a enfermeira precisa ser flexível e criativa e ainda algumas vezes enfática. A autora relata que uma família administrava grande quantidade de azeite para o recém-nascido com a finalidade de curar a icterícia. Mesmo sem respaldo da literatura do cuidado profissional, a autora manteve o uso do azeite, mas para se resguardar profissionalmente ela teve que ser enfática tentando chegar a um acordo para diminuir a quantidade de várias colheres de sopa para algumas gotas.

Em um trabalho de conclusão de especialização ${ }^{(14)}$, a autora fez a implementação do referencial de Leininger a famílias que tinham filhos lactentes, na periferia urbana com precárias condições de vida. A enfermeira refere que, para fazer a acomodação, realizam-se ações para adaptar, negociar, ou ajustar hábitos de saúde com as famílias.
Para que isso ocorra o diálogo é básico. No exemplo que a autora apresenta, fica claro que a negociação envolve o diálogo, o que possibilita a compreensão da enfermeira sobre os cuidados prestados pela família, bem como a compreensão da família sobre os cuidados propostos pela enfermeira.

$\mathrm{Na}$ dissertação de mestrado ${ }^{(15)}$ a autora realizou uma pesquisa com famílias teuto gaúchas e conclui que as ações de acomodação/negociação representam maneiras de negociar, adaptar ou ajustar os padrões de cuidado à saúde do cliente.

Pesquisas tendo como base a teoria de Leininger

A pesquisa ${ }^{(16)}$ que teve como objetivo identificar padrões de cuidado do povo ukraniano com relação a gravidez e nascimento, verificou que os significados e experiências de cuidado estão interligados com a religião, a família e a comunidade, e principalmente com as obrigações familiares, presença, suporte, intimidade e com o papel feminino. Há diversidade entre os cuidados dispensados aos jovens e idosos.Com base nestes achados, a autora propõe que, para a enfermagem acomodar/negociar o cuidado com os ukranianos, há necessidade de despender mais tempo com a mãe e com toda a família. 0 cuidado deve ser dispensado na perspectiva de ajuda ao outro, fazendo-se presente nas necessidades, mantendo mães e recém-nascidos juntos, dando informações profissionais sobre a gravidez.

$O$ estudo ${ }^{(17)}$ com americanos de origem mexicana teve 0 objetivo de conhecer os valores, crenças e práticas do cuidado e verificar se as formas de intervenção da teoria transcultural são adequadas para prover cuidado congruente numa designada comunidade. Verificou que os valores de cuidado estavam relacionados com: obter e mostrar respeito, envolvimento com a família, onipotência de Deus, amor filial, atenção para assistência direta. Além disso, para a população estudada, saúde é quase tudo, saúde é bem-estar. A autora refere, que para acomodar ou negociar o cuidado para esta população, deve haver confiança e respeito, deve-se oportunizar o envolvimento da família no cuidado direto, manter flexibilidade no horário de visitas, negociar valores de cuidado da comunidade que não poderiam ser efetivos procurando substituílos por práticas apresentadas pelos profissionais, facilitar o uso da oração junto com as práticas de rotina da enfermagem, acomodar (oportunizar) a participação de curandeiros populares.

Outro estudo ${ }^{(18)}$ se constituiu em uma pesquisa com a ordem dos antigos menonitas que se situam nos Estados Unidos e no Canadá, e que é considerada uma etnoreligião. Seus integrantes vestem roupas próprias, falam um dialeto alemão, resistem às mudanças familiares, têm rejeição à educação formal e vivem da agricultura. Esta autora, verificou que os modos de decisão de cuidado 
são diretamente influenciados pela visão de mundo, pela estrutura social e pelo contexto ambiental. Dessa forma, ela recomenda que as enfermeiras, ao cuidarem destes clientes no hospital, precisam levar em consideração a forma de vida deste grupo, acomodando o horário de visitas. Devem levar em conta suas técnicas de educação em saúde, uma vez que rejeitam o uso de televisão, e oportunizar que seus curandeiros possam penetrar no hospital para complementar o tratamento.

A pesquisa ${ }^{(19)}$ entre viúvas gregas residentes no Canadá, teve como objetivo descrever e explicar o significado das experiências de viúvas gregas idosas com respeito ao seu cuidado em saúde e ao fenômeno do luto. Verificou que os imigrantes gregos preservam valores religiosos, têm forte respeito à herança cultural, sacrificam seus bens em favor dos outros. Para as viúvas do estudo, o cuidado significa responsabilidade, reciprocidade, preocupação, amor, companhia, proteção da família, hospitalidade e ajuda derivada de sua rede social e religião. Elas também procuram manter o estado de bem-estar realizando atividades diárias e evitando dor e doença. Para fazer a acomodação/negociação de cuidado, as enfermeiras precisam compreender como a família se mantinha antes e como se manterá financeiramente no futuro, ouvir e respeitar a relutância das viúvas em compartilhar os aspectos negativos do marido falecido, ter tempo para estabelecer confiança, aprender sobre sua religião, descobrir as relações familiares e aprender sobre suas práticas de saúde populares.

No estudo ${ }^{(20)}$ realizado entre um grupo de ciganos em São Paulo a autora refere na explicitação de seu referencial teórico, que acomodação são formas de adaptação, negociação ou ajustamento aos hábitos de saúde e de vida dos indivíduos e clientes. Como resultado, refere que, embora o cigano tenha seus hábitos e conhecimentos fortemente marcados e dominados pelo chefe, poderia ser tentada uma negociação para a diminuição de pimenta na alimentação, bem como do fumo, visando diminuir a alta incidência de problemas de úlcera e problemas cardíacos. A autora, no entanto, não fornece dados sobre como se faria esta negociação.

\section{Enfocando os atributos}

A partir das características do conceito que apareceram ao longo da literatura, realiza-se a categorização, chegando-se, assim, aos atributos, ou seja, às qualidades inerentes ao mesmo ${ }^{(6)}$. Neste trabalho foi feita a categorização dos cuidados apresentados pelos autores, que ora se apresentavam de forma explícita como elementos do cuidado; ora estavam implícitos, quando o autor descrevia como realizou os cuidados, ou no caso das pesquisas, o que os entrevistados consideravam importante no cuidado.

Assim, para o conceito negociar, chegou-se aos seguintes atributos: adaptar, ajustar, interação verbal em que as partes propõem e contrapõem, buscar entendimento, celebrar. Baseada nesta categorização, considero que negociar é ajustar os modelos explicativos do cliente/família e do profissional acerca do cuidado, no qual as partes propõem e contrapõem utilizando recursos para buscar o entendimento.

Para acomodar, chegou-se aos seguintes atributos: negociar, ajustar, oportunizar, oferecer condições, adaptar, unir, conjugar. Para acomodar, a enfermeira deve oferecer condições para conjugar os modelos explicativos do cliente e do profissional.

Antecedentes

Antecedentes são aqueles eventos que precisam ocorrer para possibilitar a ocorrência do conceito ${ }^{(6)}$. Na literatura geral, encontramos que, para ocorrer a negociação, cada parte precisa entrar com recursos e necessidades, possibilitando o poder de barganha de ambas as partes. Na literatura de enfermagem transcultural, emergiu a questão do diálogo, partir da experiência do outro. Deve também haver flexibilidade e criatividade da enfermeira.

Para ocorrer a acomodação é preciso negociar, sugerir, trocar idéias, facilitar, auxiliar, oferecer condições para. Estes dados que emergiram da literatura da enfermagem transcultural levam a entender que é a enfermeira que criará estas condições.

E para a acomodação/negociação é preciso dialogar, despender mais tempo com o cliente, dar suporte, fazer-se presente nas necessidades, confiar, respeitar, oportunizar, levar em consideração, compreender, ouvir.

Conseqüências

São aqueles eventos que resultam da ocorrência do conceito $^{(6)}$.

De acordo com a literatura geral, tanto a acomodação como a negociação têm como conseqüência resolver conflitos ou divergências ${ }^{(8)}$, e a negociação visa 0 acordo ${ }^{(10)}$. Na literatura da enfermagem transcultural, Leininger e seus seguidores esperam alcançar a congruência do cuidado entre o modelo explicativo da cliente/família e do profissional.

Oposição aos conceitos de negociação/acomodação

A imposição cultural é a tendência dos profissionais de saúde imporem suas crenças, valores e práticas sobre outra cultura porque acreditam que somente suas idéias são as verdadeiras, portanto superiores às do outro grupo ${ }^{(21)}$. Isto é, ocorre a imposição do modelo explicativo do profissional de saúde sobre o modelo explicativo do cliente/família. 


\section{COMENTÁRIOS FINAIS}

0 grande desafio das enfermeiras e dos enfermeiros que se aventuram pelos caminhos da antropologia para cuidar do ser humano de uma forma mais abrangente é, certamente, realizar o olhar antropológico. Isto é, dentro da cultura da enfermagem, tirar por momentos a armadura do modelo profissional e ver o cliente no seu mundo, entender suas ações, mas ao mesmo tempo não deixar de ser o enfermeiro ou a enfermeira. Como ser um e outro ao mesmo tempo?

A teoria de Leininger é uma proposta para vencer este desafio. Porém, por ser uma teoria abrangente, tem conceitos abstratos, havendo assim um amplo campo para dar mais respostas ao desafio de como utilizar esta visão antropológica na enfermagem.

No que se refere à comparação entre acomodação e negociação, pude observar que nas definições do dicionário ${ }^{(8)}$ há pontos de encontro. Porém, pode-se inferir que a negociação leva a uma idéia mais dinâmica de ação, enquanto que a acomodação dá idéia de enquadramento, algo passivo. Ainda na literatura geral ${ }^{(9)}$, considera-se que a negociação é uma ação na qual se propõem e contrapõem e que, para haver negociação, cada parte traz recursos e necessidades a serem satisfeitas. Alerta ainda que, se estes recursos não forem equivalentes, não haverá negociação e sim imposição. Cada parte tem percepções e expectativas de si e do outro na hora de negociar.

Eu pondero, após realizar esta análise, que a negociação é diferente da acomodação, e que se faz mais acomodação do que negociação. Fazer negociação é algo difícil, pois os recursos que são trazidos para esta ação seriam básicos para manter o poder de barganha, possibilitando a cada parte propor e contrapor. Em estudo ${ }^{(22)}$ sobre a negociação da participação dos pais no cuidado da criança hospitalizada, os autores referem que, apesar das enfermeiras se proporem a negociar, isso se torna difícil, pois os recursos dos pais são inferiores, haja vista que estão num ambiente estranho, estão ansiosos, passando por uma situação difícil, com muitas incertezas, enquanto que as enfermeiras estão em seu ambiente usual, com o controle das informações, o que aumenta 0 seu poder de barganha.

$\mathrm{Na}$ literatura transcultural anteriormente apresentada, a questão do controle do cliente não é mencionada, já que a própria teoria não a contempla. Pensando nas diferenças de classe, de gênero, de nível educacional existente no Brasil, onde o acesso ao prontuário é vedado a qualquer cliente, podemos inferir que o controle e, como conseqüência, a negociação estão distantes do cotidiano. Nas pesquisas apresentadas, observa-se que a acomodação se sobressai, pois é a enfermeira que vai "oportunizar" o horário de visitas, compreendendo o modo de vida de determinada cultura, que vai facilitar, que irá ouvir ou respeitar. De qualquer maneira, é a enfermeira e não o cliente, que na acomodação, possui o controle da situação.

$\mathrm{Na}$ literatura relacionada à aplicação dos referenciais teóricos, apenas um estudo ${ }^{(13)}$ que mais se aproxima da negociação, quando refere que, durante a assistência, garantia uma forma participativa de diálogo partindo das experiências das mulheres. Apesar dessa tentativa, é flagrante que o controle da situação estava com a enfermeira; era ela quem garantia a forma de participação. Além disso, devemos nos perguntar como estas mulheres se sentiam diante da enfermeira procedente de outra classe social? Críticos da Teoria de Leininger acreditam que seu foco na cultura omite variáveis como o gênero e a classe social ${ }^{(23)}$.

Há ainda o argumento de que o modelo de negociação da Teoria de Leininger é criticado porque as enfermeiras podem potencialmente manipular mais do que negociar o cuidado ${ }^{(24)}$. Em outras palavras, as enfermeiras usam seu conhecimento sobre a visão do cliente para conseguir o que elas querem que o cliente faça. Isso é reforçado por outro estudo ${ }^{(25)}$, que à luz dos conceitos de Foucault sobre o poder, fez análise de duas dissertações de mestrado que utilizaram a teoria na prática com famílias. Verificou-se que, ao compreender a visão de mundo do sistema familiar de cuidado, a enfermeira age com mais poder disciplinador, interferindo assim, na vida da família.

No Brasil, a teoria de Leininger tem aproximado mais e mais as enfermeiras da antropologia. Sem dúvida, utilizar uma teoria com base na antropologia e na enfermagem é uma contribuição para aproximar o mundo do cliente do mundo do profissional da saúde e fomentar o conhecimento na enfermagem. Para que isso ocorra, devemos estar atentos para que não façamos uma aplicação da teoria de forma repetitiva, sem avaliações críticas, incorrendo no risco de gastar energias em vão. A teoria de Leininger, bem como outras abordagens com base na antropologia, tem limitações, e, como mostra a análise deste trabalho, tem ambigüidades, termos que precisam ser clarificados dentro do contexto onde serão aplicados.

Para concluir, é importante enfatizar que, com as experiências já existentes na aplicação desta teoria na prática e na pesquisa, à luz da literatura crítica que vem despontando, urge que as enfermeiras, ao se definirem em trabalhar com esta teoria ou outra abordagem que tenha base na antropologia, busquem avançar no sentido de pensar esta abordagem dentro do contexto real, local e nacional. Que as outras formas de efetivar o cuidado preconizadas na teoria, que são a preservação e a repadronização, sejam também clarificadas. Ainda, é importante enfatizar que este trabalho, com suas limitações, pretende ser apenas um início para outros trabalhos. 


\section{REFERÊNCIAS BIBLIOGRÁFICAS}

1. Uchoa E, Vidal JM. Antropologia médica: elementos conceituais e metodológicos para uma abordagem da saúde da doença. Cad Saúde Pública 1994 out/dez;10(4):497-504.

2. Leininger M. Transcultural nursing. New York: John Wiley; 1978.

3. Leininger M. Culture care diversity and universality: a theory of nursing. New York: National League for Nursing; 1991a.

4. Boehs AE. Prática do cuidado ao recém nascido e sua família, baseada na teoria transcultural de Leininger e na teoria do Desenvolvimento da Família. [dissertação]. Florianópolis (SC): Pós Graduação em Enfermagem/UFSC; 1990.

5. Meleis A. Strategies for concept development. In: Meleis, A. Theoretical nursing: development and progress. Philadelphia: J. B. Lippincott; 1997. p.203-22

6. Walker LO, Avant KC. Strategies for theory construction in Nursing. Norwalk (CT): Appleton \& Lange; 1995.

7. Morse J. Exploring the theoretical basis of nursing using advanced techniques of concept analysis. Adv Nurs Sci 1995 Mar; 17(3):31-46. 8. Moderno dicionário da língua portuguesa Michaelis. São Paulo: Melhoramentos; 1998.

9. Zajdsznajder L. Teoria e prática da negociação. Política da negociação. 2.ed. Rio de Janeiro: José Olímpio;1988.

10. Patrício ZM. A prática do cuidar/cuidado à família da adolescente grávida solteira e seu recém nascido. [dissertação]. Florianópolis (SC): Pós Graduação em Enfermagem/UFSC; 1990.

11. Koeller E. Cuidando de famílias sob o impacto do vírus HIV em seu espaço sócio-cultural. [dissertação]. Florianópolis (SC): Pós Graduação em Enfermagem/UFSC; 1992.

12. Castanhel $M, B o e h s ~ A E$. Cuidando de uma família na comunidade - uma experiência utilizando um referencial teórico. Texto Contexto Enfermagem 1993 jul/dez; 2(2):87-98.

13. Monticelli M. Nascimento como um rito de passagem: abordagem para 0 cuidado às mulheres e recém-nascidos. São Paulo: Robe Editorial; 1997.
14. Souza MA. Cuidando da família em expansão na comunidade do Sol Nascente.[Trabalho de Conclusão de Curso, Especialização de Enfermagem da Família]. Florianópolis (SC):Pós Graduação em Enfermagem/UFSC; 1996.

15. Schwartz E. Família teutogaúcha: o cuidado entre as possibilidades e limites. [dissertação]. Florianópolis (SC): Pós Graduação em Enfermagem/UFSC; 1998.

16. Bohay IZ. Culture care meanings and experiences of pregnacy and childbirth of ukrainians. In: Leininger M. Culture care diversity and universality: a theory of nursing. New York: National League for Nursing; 1991. p.179-201.

17. Stasiak DB. Culture care theory with mexican-american in an urban context. In: Leininger M. Culture care diversity and universality: a theory of nursing. New York: National League for Nursing; 1991. p.20329.

18. Wenger AF. The cultural care theory and the old order amish. In: Leininger M. Culture care diversity and universality: a theory of nursing. New York: National League for Nursing; 1991. p.147-78.

19. Rosenbaum J. Cultural care theory and greek canadian widow. In: Leininger M. Culture care diversity and universality: a theory of nursing. New York: National League for Nursing; 1991. p.305-29.

20. Braga CG. Enfermagem transcultural e as crenças valores e práticas do povo cigano. Rev Esc Enfermagem USP 1997 dez; 31(3):498-516.

21. Leininger M. Becoming aware of types of health practioners and cultural imposition. J Transcultural Nurs 1991b. Dec/Feb; 2(2):32-9.

22. Callery P, Smith L. A study of role negociation between nurses and the parents of hospitalized children. J Adv Nurs 1991;16:772-81. 23. Wilkins $H$. Transcultural nursing: a selective review of the literature, 1985-1991. J Adv Nurs 1993; 18:602-12.

24. Lea A. Nursing in today s multicultural society: a transcultural perspective. J Adv Nurs 1994; 20:307-13.

25. Boehs AE. Que poder a enfermeira utiliza na abordagem cultural do cuidado à família? Rev Enfermagem UERJ 1999 jan/jun; 7(1):617. 